\title{
Spirometer-controlled cine magnetic resonance imaging used to diagnose tracheobronchomalacia in paediatric patients
}

\author{
Pierluigi Ciet ${ }^{1,2}$, Piotr Wielopolski ${ }^{1}$, Rashindra Manniesing ${ }^{3}$, Sandra Lever $^{2}$, \\ Marleen de Bruijne ${ }^{4,5}$, Giovanni Morana ${ }^{6}$, Pier Carlo Muzzio7, Maarten H. Lequin ${ }^{1}$ \\ and Harm A.W.M. Tiddens ${ }^{1,8}$

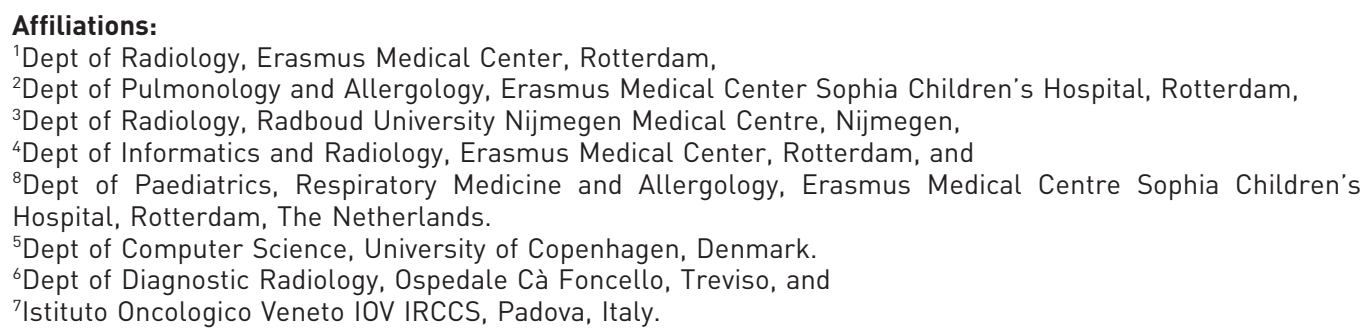

Correspondence: H.A.W.M. Tiddens, Erasmus Medical Centre Sophia Children's Hospital, Depts of Pediatrics, Respiratory Medicine and Allergology, and Radiology, P.O. Box 2060, 3000 CB Rotterdam, The Netherlands. E-mail: h.tiddensđerasmusmc.nl

ABSTRACT Tracheobronchomalacia (TBM) is defined as an excessive collapse of the intrathoracic trachea. Bronchoscopy is the gold standard for diagnosing TBM; however it has major disadvantages, such as general anaesthesia. Cine computed tomography (CT) is a noninvasive alternative used to diagnose TBM, but its use in children is restricted by ionising radiation. Our aim was to evaluate the feasibility of spirometer-controlled cine magnetic resonance imaging (MRI) as an alternative to cine-CT in a retrospective study.

12 children with a mean age (range) of 12 years (7-17 years), suspected of having TBM, underwent cineMRI. Static scans were acquired at end-inspiration and expiration covering the thorax using a threedimensional spoiled gradient echo sequence. Three-dimensional dynamic scans were performed covering only the central airways. TBM was defined as a decrease of the trachea or bronchi diameter $>50 \%$ at endexpiration in the static and dynamic scans.

The success rate of the cine-MRI protocol was $92 \%$. Cine-MRI was compared with bronchoscopy or chest CT in seven subjects. TBM was diagnosed by cine-MRI in seven (58\%) out of 12 children and was confirmed by bronchoscopy or CT. In four patients, cine-MRI demonstrated tracheal narrowing that was not present in the static scans.

Spirometer controlled cine-MRI is a promising technique to assess TBM in children and has the potential to replace bronchoscopy.

@ERSpublications

Spirometer controlled cine-MRI: a technique to assess TBM in children with the potential to replace bronchoscopy/cine-CT http://ow.ly/pDNAA

\footnotetext{
This article has supplementary material available from www.erj.ersjournals.com

Received: July 062012 | Accepted after revision: April 012013 | First published online: April 182013

Conflict of interest: None declared.

Copyright @ERS 2014
} 


\section{Introduction}

Tracheobronchomalacia (TBM) is defined as an excessive collapse of the intrathoracic part of the trachea and/or main bronchi during expiration [1]. When TBM is suspected, a diagnostic bronchoscopy, which is the current gold standard, is used to confirm the diagnosis $[1,2]$. In paediatric populations, bronchoscopy has the disadvantage that it is invasive and requires general anaesthesia, while the child maintains spontaneous breathing [1]. In addition, relevant daily life breathing manoeuvres, such as forced expiration and coughing, cannot be routinely performed during the procedure [3-4]. Bronchoscopy using conscious sedation, which preserves spontaneous ventilation for the evaluation of TBM, is used in adults [4]. However, this technique in children is, in general, not feasible due to a lack of cooperation [4]. Finally, bronchoscopy does not provide exact measurements of airway dimensions comparable to that provided by imaging techniques [3-5].

For these reasons an alternative to bronchoscopy, cine computed tomography (CT), has been used to assess TBM [6]. An advantage of this technique is that the collapsibility of trachea and mainstem bronchi can be evaluated during static and dynamic conditions [7-9]. However cine-CT to detect TBM exposes the patient to ionising radiation [1], which is a more significant problem for children than adults because they are more susceptible to the harmful effects of ionising radiation [10-14]. Cine-magnetic resonance imaging (MRI) might be an attractive, radiation-free alternative to cine-CT to diagnose TBM [15-19]. Recent improvements in MR technology (ultrafast imaging) allow dynamic evaluations of the central airway dimensions (cine-MRI) [15]. Ideally, breathing manoeuvres during cine-MRI should be standardised using cine-MRI spirometry. A MRI compatible spirometer was recently developed and tested [20]. To our knowledge, spirometer controlled cine-MRI has not been applied before in TBM assessment $[16,18]$.

We have previously demonstrated feasibility of the spirometer-controlled cine-MRI protocol in healthy adult volunteers [21]. The aim of this study was to retrospectively evaluate the diagnostic feasibility of this protocol in a group of children, who needed a chest MRI for various indications and in whom the treating clinician requested assessment of TBM to be included in the evaluation. In this paper we describe our methodology and the results of a retrospective image analysis of static and dynamic changes in central airway dimensions in 12 patients.

\section{Materials and methods}

MRI

The cine-MRI protocol for the diagnosis of TBM was developed in 10 healthy adult volunteers mean age (range) 31 years (30-33 years) for which approval was obtained from our institutional ethics committee [21]. Scanning was performed using a 1.5T Signa Hdxt MRI (General Electric Healthcare, Milwaukee, WI, USA) and using the whole body coil for radiofrequency excitation and an eight-channel torso coil for signal reception. The protocol consisted of static and dynamic acquisitions. Three-dimensional (3D) "static" images at total lung capacity and residual volume were acquired by two 13-s breath-holds covering the complete thoracic region at end-inspiration and end-expiration using a $3 \mathrm{D}$ radiofrequency-spoiled gradient echo sequence $\left(\right.$ SPGR) with repetition time /echo time $=1.4 / 0.6 \mathrm{~ms}$, flip angle $2^{\circ}$, sagittal volume acquisition with a true isotropic $3.0 \times 3.0 \times 3.0 \mathrm{~mm}^{3}$ voxels. $3 \mathrm{D}$ cine-MRI acquisitions were acquired with the same imaging parameters and voxel resolution, but only covering the trachea and mainstem bronchi ( $12 \mathrm{~cm}$ sagitally). This was used to measure their dimensions and to monitor, in pseudo-real time, the airway dimensions to detect dynamic TBM during forced expiration and cough. The temporal resolution of $400 \mathrm{~ms}$ per volume was achieved using the temporally resolved imaging of contrast kinetics scan platform with accelerated imaging options [22]. 48 volumes were collected in $19 \mathrm{~s}$. Overall the acquisition time per patient, including localisers, adjustments, breathing instructions and executing all scans, was $\sim 15 \mathrm{~min}$ (range 9-20 min). The videos of the dynamic acquisitions are provided in the online supplementary material.

\section{Bronchoscopy}

Bronchoscopies in six out of the 11 patients were carried out by a paediatric pulmonologist as part of diagnostic workup in routine patient care, using a flexible bronchoscope, external diameter $3.5 \mathrm{~mm}$ or $5.5 \mathrm{~mm}$ (Olympus; Tokyo, Japan), during general anaesthesia while maintaining spontaneous breathing. Airway malacia was diagnosed by visual inspection of the airway shape and dynamics during spontaneous breathing without positive end-expiratory pressure, and during spontaneous coughing in case this occurred. Malacia was defined as collapse of at $\geqslant 50 \%$ of the airway lumen, during the expiration phase while spontaneous breathing or while the patient was coughing [2]. 


\section{Breathing instructions}

Paediatric patients, who needed a chest MRI for various clinical indications and in whom the treating clinician requested assessment of TBM, based on their clinical presentation, were scheduled for a spirometer controlled cine-MRI. Half an hour before the MRI all children did a routine upright spirometry, coached by a lung function technician. Next, the required breathing manoeuvres were repeated in the supine position on a stretcher using an MRI-compatible spirometer (custom made Masterscope MRI; CareFusion, Houten, The Netherlands). The purpose of the training was: 1) to monitor and standardise breathing manoeuvres by using spirometer volume data during the MRI; 2) to reduce anxiety related to MRI investigations; and 3) to increase the number of successful MRI investigations. For the static MRI acquisitions, children were trained to obtain a maximal breath-hold time of $15 \mathrm{~s}$ at $\geqslant 95 \%$ of the inspired vital capacity (VC) and at $\geqslant 90 \%$ of the expired VC. For the dynamic cine-MRI sequences, patients were asked to perform two expiration manoeuvres, both starting at $\geqslant 95 \%$ of the inspired VC. The first dynamic manoeuvre was a full forced expiration, which was done as follows. From tidal breathing, patients were asked: 1) to take a deep breath and reach $\geqslant 95 \%$ of inspired $\mathrm{VC} ; 2$ ) to hold their breath for a few seconds; 3 ) to perform a forced expiration and reach $\geqslant 90 \%$ of the expired VC; 4 ) to hold their breath for a few seconds; and 5) to breathe quietly i.e. tidal volume breathing. The second dynamic manoeuvre was a full expiration manoeuvre while the patient produced a series of repeated coughs starting at $\geqslant 95 \%$ of inspired VC $\geqslant 90 \%$ of the expired VC.

After the training session, the patient was transferred to the MRI scanner. Breathing instructions during the MRI were given by the same lung function technician that performed the training, using the MRI compatible built-in patient audio system. During the MRI investigation, the lung technician sitting next to the MRI technician monitored the inspired and expired volumes and dynamic flows on the computer screen of the MRI-compatible spirometer set-up (fig. 1). Based on the spirometer data, the lung function technician instructed the MRI technician on when to start or stop the scans in case of patient error.

\section{Image analysis}

The first post-processing step for the lung and central airway images was performed on the scanner console and consisted of equalising the inhomogeneity/signal attenuation of the receiver array coil by using a surface coil correction intensity algorithm and adaptive filtering to enhance the signal-to-noise ratio. Image quality criteria were defined for both static and dynamic scans. Static scans were repeated if blurring of structures and ghosting were observed. Dynamic scans were repeated when excessive blurring and ghosting of the chest wall was observed. The latter was indicative of a lack of temporal resolution or due to a suboptimal breathhold, as shown on the spirometer screen.

The image data were then transferred to an Advantage windows workstation, or onto the Advantage Windows Server (General Electric Healthcare) to perform cine multi-planar reformats (MPR) from the 3D cine-MRI data collected. The MRIs were jointly evaluated by two radiologists (P. Ciet and M.H. Lequin)
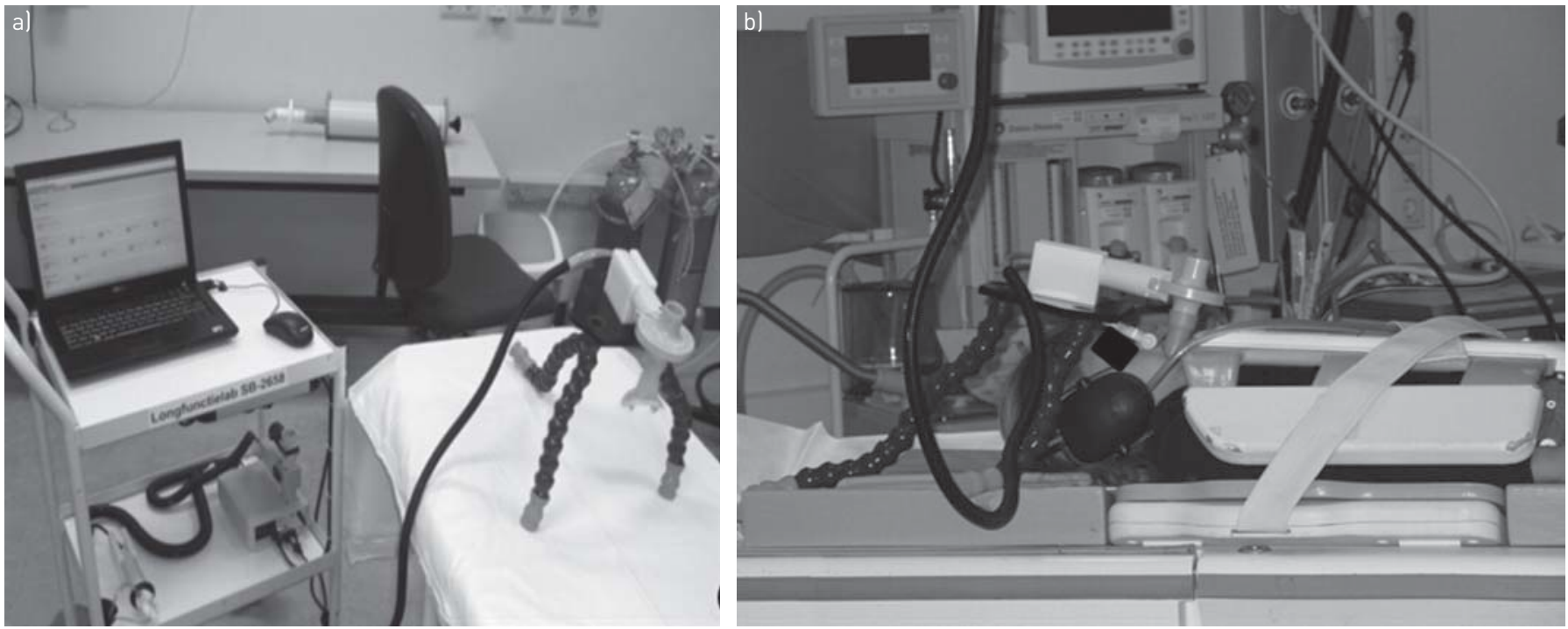

FIGURE 1 Magnetic resonance imaging (MRI) spirometer set-up. a) Metal-free plastic tripod, mouthpiece and the MRI compatible spirometer in the lung function room where the breathing manoeuvres were trained. b) In the MRI suite, the spirometer is connected to the computer by long plastic tubing, which is introduced into the Faraday cage, to measure differential pressure for computation of flow. 
and a standard report was written. For the retrospective analysis, all images were assessed by a radiologist (P. Ciet) blinded to all clinical data using manual tracing as described below.

The manual assessment started by analysing both the end-inspiration and end-expiration static volumes in the four-dimensional (4D) MPR platform. Reformats were performed to measure the diameters of the trachea and main stem bronchi using a window and magnification setting (zoom factor 3.0). Windowing was performed by setting the window level at one half of the maximum signal recorded around the trachea with a window width of 0 . First, the narrowest section of the trachea in expiration was determined using a two-dimensional (2D) calliper to measure the anteroposterior diameter in a view perpendicular to the trachea midline (fig. 2). To compare the degree of narrowing, a similar section, angled according to the main tracheal axis, was reformatted using the surrounding anatomical structures as spatial reference and the distance to the carina. Next, the dynamic acquisitions (both forced expiration and coughing) were evaluated in cine-mode on the $4 \mathrm{D}$ reconstruction platform both in longitudinal and perpendicular views of the trachea to determine which one of the 48 volumes showed the greatest tracheal collapse (fig. 3 ). Having

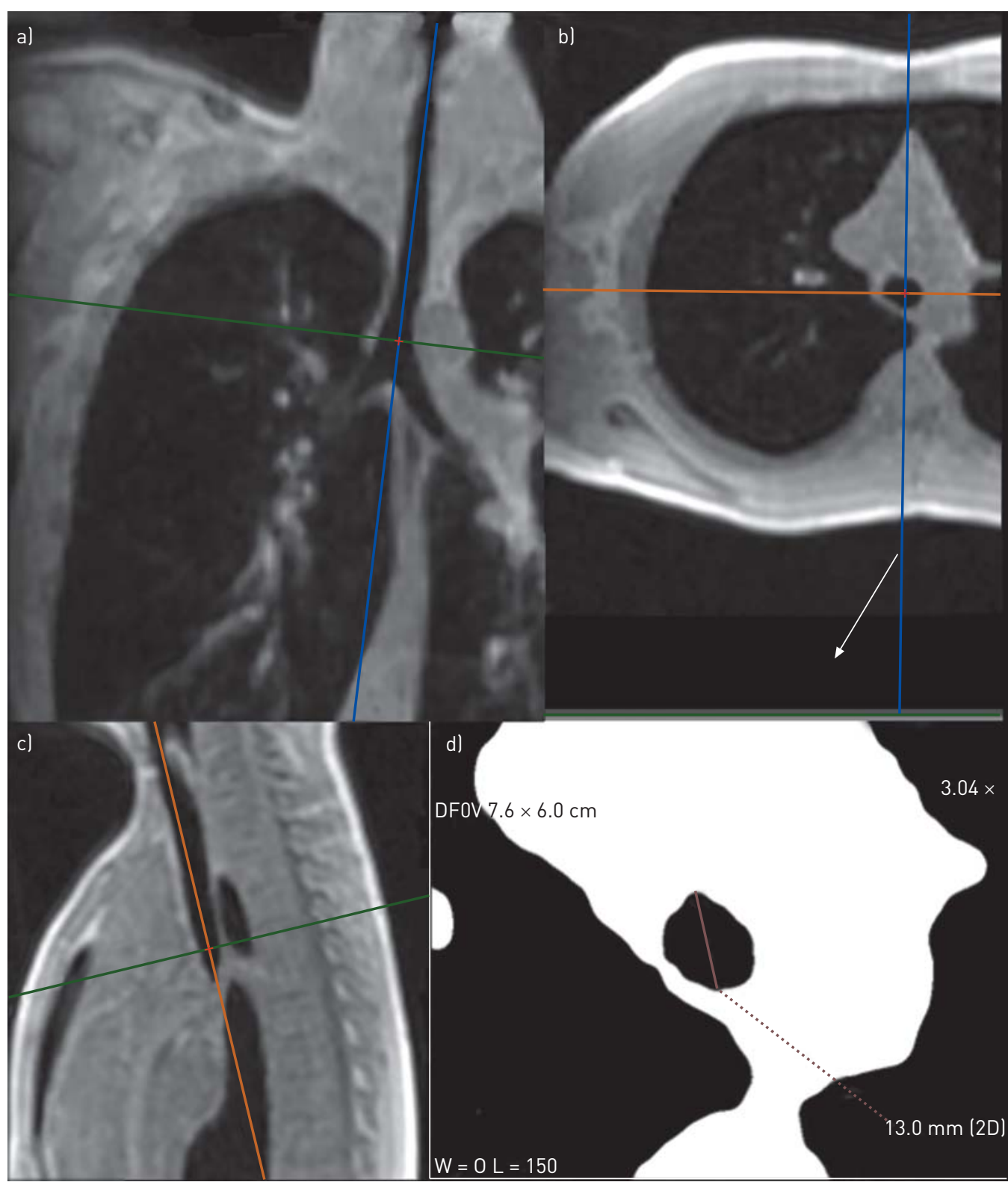

FIGURE 2 Image analysis of dimensions for the trachea in patient B. a-c) Reformats of three-dimensional radiofrequency-spoiled gradient echo sequence breath-hold during inspiration. In part a) blue line and in part c) the orange line indicates the correct orientation along the midline of the trachea. d) Shows the anteroposterior diameter in reformatted axial view (double oblique) and with the window set at full-width half-maximum image. 


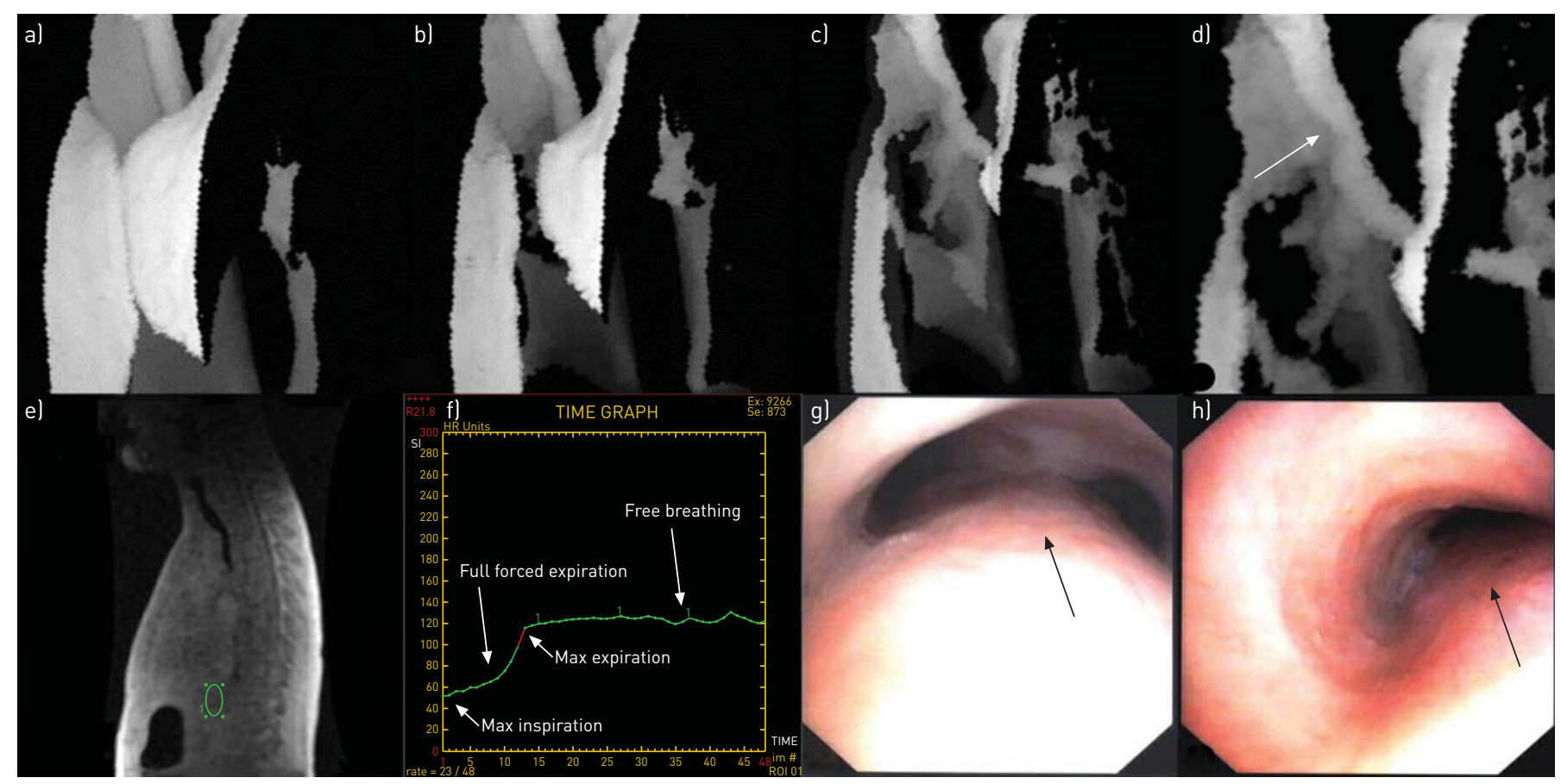

FIGURE 3 Dynamic magnetic resonance imaging (a-e), respiratory waveform (f) and bronchoscopy images (g, h) in patient C. a-d) Three-dimensional volume rendering at different time points during the forced expiration manoeuvre. Note the trachea impression above the carina (d) indicated by a white arrow. e) Sagittal view with green region of interest to assess signal intensity changes according to the breathing cycle. f) Correspondent signal intensity changes (approximate respiratory waveform) over time, where y-axis represents signal intensity and x-axis the time. Note the inverted relationship between volume of air in the lungs and signal intensity. The patient started the forced expiratory manoeuvre from maximum inspiration, which showed the lowest signal intensity, until maximum expiration, which had the greatest signal intensity. g, h) Screen shots of the bronchoscopy evaluation in the same patient show bulging of the pars membranacea (black arrows) in addition to the anterior impression (h) just above the carina caused by the innominate artery, which corresponds to the tracheal impression as shown on image (d).

selected the "most collapsed" volume, the same distance measurements were performed as described for the breath-hold acquisitions.

TBM was defined as a reduction in airway anteroposterior diameter of the trachea of $>50 \%$ between static end-inspiration and end-expiration scans or between end-inspiration and dynamic scans as routinely used in the bronchoscopy assessment of TBM [17]. This definition was used to compare the diagnosis of TBM using cine-MRI with the subjective diagnosis made by the physician for those patients in whom a bronchoscopy was performed.

\section{Comparison between MRI and bronchoscopy or CT}

In six subjects bronchoscopy reports were available, which were compared to $3 \mathrm{D}$ cine-MRI. Since, using bronchoscopy, TBM is diagnosed by visual assessment and not by exact measurements of airway dimensions, bronchoscopy reports were used to verify the 3D cine-MRI findings. In one subject, only a spirometer controlled CT was available for confirmation of the diagnosis of TBM. For this patient, chest CT images were compared to cine-MRI findings.

\section{Results}

Our retrospective analysis included 12 paediatric patients (table 1), who all had a cine-MRI made for various clinical diagnostic reasons. Informed consent was obtained from the parents of the patient for anonymous analysis of the data and registered in the electronic patient record. Approval for this retrospective study was obtained from the institutional review board.

The success rate of the MRI protocol was 92\%, only one participant was not able to successfully complete all required breathing manoeuvres, because of fear induced by the noise in the MRI scanner. Images were of diagnostic quality for analysis in all subjects, except for the patient mentioned above. Airway dimensions measured by the manual assessment are displayed in table 1. TBM was diagnosed in seven children (58\%): patient B, C, D, F, L, M and N (table 1). In patients B and C, TBM was visible on the static and dynamic MRI images, whereas in patients D, F, L, M and N, TBM was visible only in the dynamic MRI acquisitions. Patient B and $\mathrm{N}$ had severe TBM, which involved the trachea at the level of the carina and the left main 


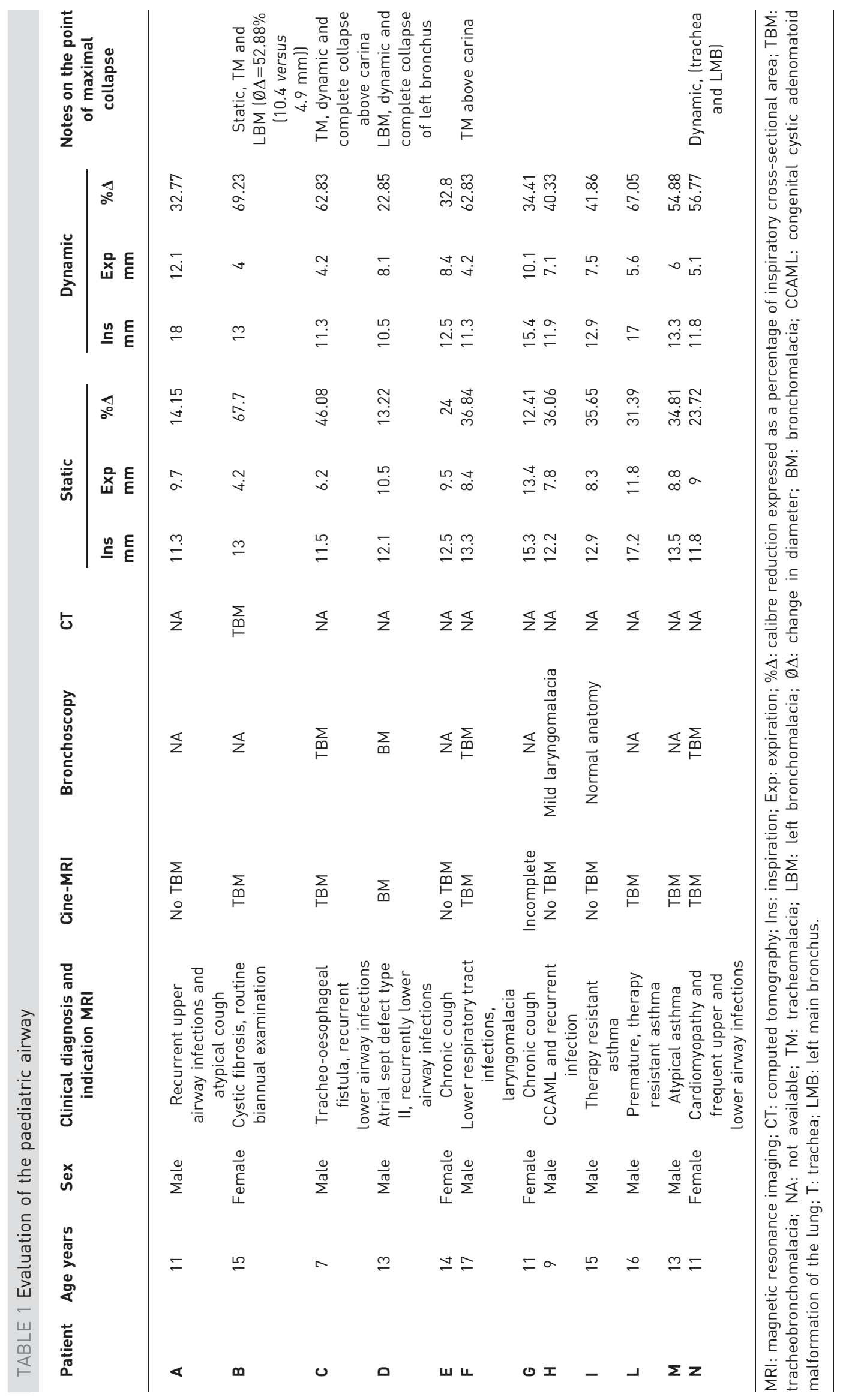




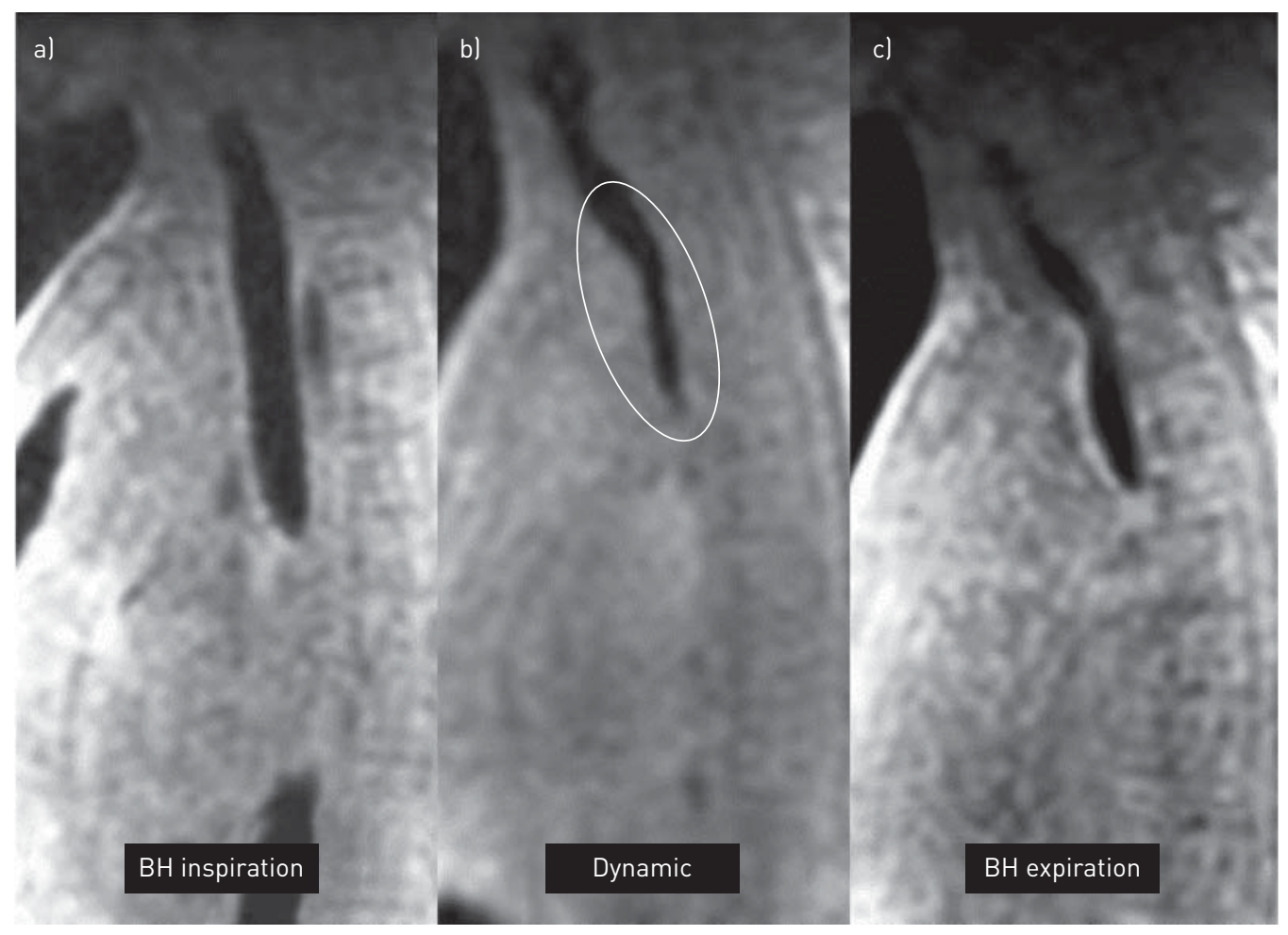

FIGURE 4 Tracheobronchomalacia in patient F. Breath-hold (BH) inspiration (a), dynamic forced expiration manoeuvre (b) and $\mathrm{BH}$ expiration (c). Note the trachea lumen in the dynamic acquisition is smaller than the breath-hold expiration acquisition (white oval).

bronchus. In patient B, who declined bronchoscopy, a follow-up chest CT scan confirmed the diagnosis of TBM. Patient C had TBM $1 \mathrm{~cm}$ above the carina, confirmed by bronchoscopy (fig. 3). Patient D had complete collapse of the left, main bronchus during forced expiration, which was defined as "isolated distal narrowing" by bronchoscopy. Patient F had a severe TBM, just above the carina, clearly visible during forced expiration, which was confirmed by bronchoscopy evaluation (figs 4 and 5). Patients L and M had TBM $\sim 1.5 \mathrm{~cm}$ above the carina bifurcation. Patients $\mathrm{H}$ and I had negative results for TBM both on MRI and bronchoscopy assessments. Patients A, E, and G were negative for TBM to cine-MRI assessment and no further diagnostic procedures were felt necessary by the treating physician. Patients $\mathrm{L}$ and $\mathrm{M}$ had positive results for TBM by cine-MRI assessment, and further assessment by bronchoscopy was proposed by the treating physician but declined by the parents. Hence, for all seven patients with available bronchoscopy and/or CT, cine-MRI was in concordance with bronchoscopy and/or CT for the presence or absence of TBM. For four patients this could not be evaluated.

\section{Discussion}

Our retrospective study shows that spirometer controlled 3D cine-MRI is a promising technique in children for the static and dynamic evaluation of central airway dimensions and could potentially replace bronchoscopy for the evaluation of TBM.

\section{Cine-MRI versus bronchoscopy}

The most important advantage of 3D cine-MRI was that airway dimensions could be studied during standardised static and dynamic breathing manoeuvres, such as forced expiration and cough. The use of a MRI compatible spirometer allowed us to monitor inspiratory and expiratory flows and volume during the MRI acquisitions [20]. By acquiring simultaneous functional and morphological data, the severity of the TBM can be established in a standardised fashion.

To evaluate airway dimensions using bronchoscopy in a child, general anaesthesia is frequently needed. The challenge for the anaesthesiologist is to control the level of anaesthetic in such a way that the child continues to breathe spontaneously. In instances where the anaesthesia is not deep enough, the patient begins to move and often coughs, a situation that the anaesthesiologist tries to avoid. In cases where the anaesthesia is too deep or when a muscle relaxant is used, spontaneous breathing stops and positive-pressure ventilation is 

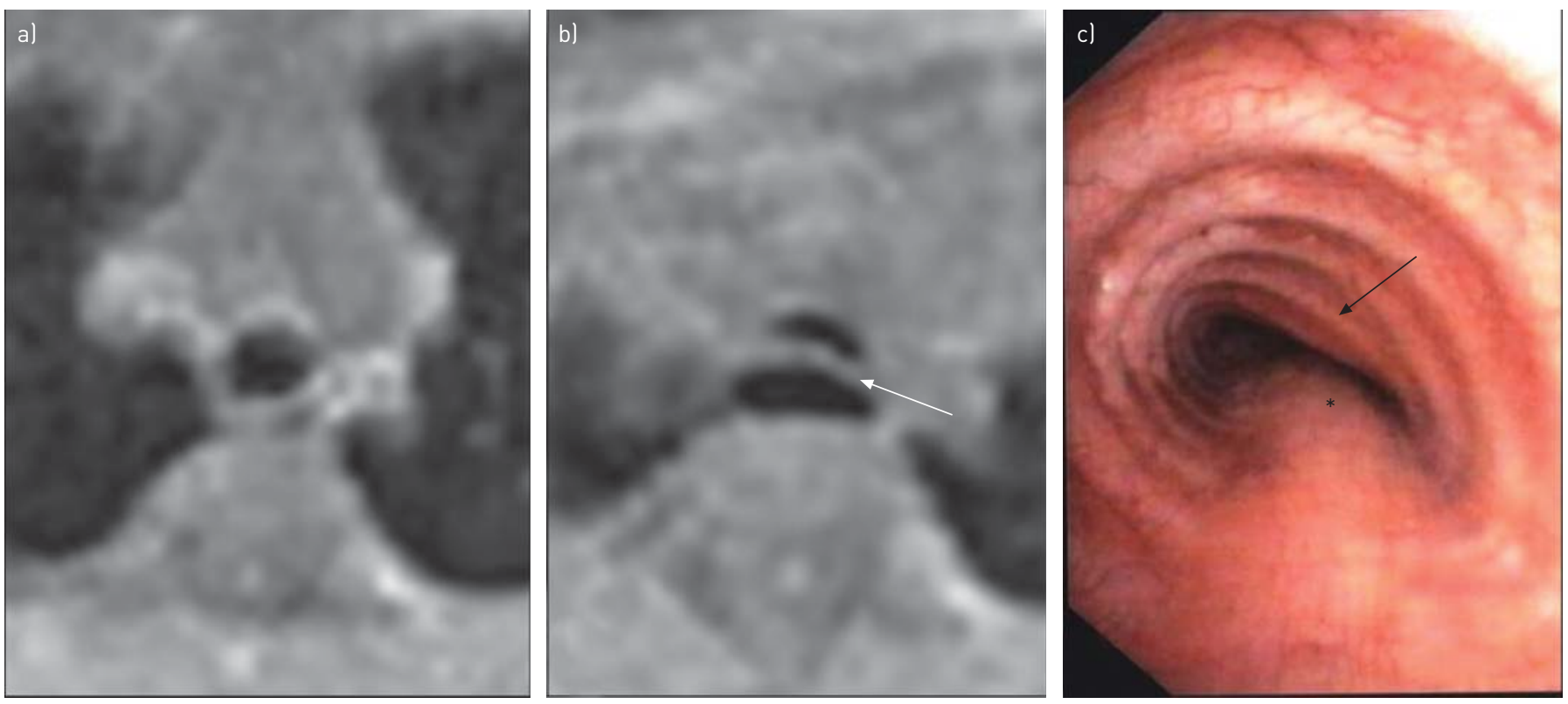

FIGURE 5 Axial reformatted images of patient F. a) Breath-hold magnetic resonance image at end inspiration showing a normal round configuration of the trachea. b) Breath-hold at end expiration at the same level, note that the pars membranacea is bulging into the lumen (white arrow). c) Shows the same location as a) and b) during bronchoscopy evaluation during tidal volume breathing, which confirms tracheomalacia caused by an abnormal configuration of cartilage rings (black arrow) combined with bulging of the pars membranacea $\left(^{*}\right)$.

needed, which can obscure TBM due to the positive trans-pulmonary pressure. Conscious sedation enables controlled breathing manoeuvres during flexible bronchoscopy to be attempted. However, in children, this technique is not used routinely since it is highly dependent on cooperation, which is considered challenging for most children [4]. Hence, cine-MRI in children aged $\geqslant 8$ years is an attractive alternative for bronchoscopy to study airway dimensions using standardised breathing manoeuvres.

The second advantage of cine-MRI is that it allowed us to obtain objective airway dimensions using postprocessing image analysis tools. This is complicated to do with bronchoscopy and, therefore, not routinely performed [5]. Despite developments in bronchoscopy optics, the image remains essentially 2D and distorted [5]. Hence, the diagnosis of malacia using bronchoscopy is mostly subjective and not very reproducible [5]. Importantly MRI has multiplanar imaging capabilities, so 3D data sets can be reconstructed providing measurements in every possible plane and cross-section $[16,18]$.

The third advantage of 3D cine-MRI is that the procedure is less invasive, relative to bronchoscopy, since it does not require general anaesthesia and anaesthesia related risks can be avoided. Bronchoscopy under general anaesthesia introduces a small risk of complications, such as respiratory depression, oxygen desaturation, apnoea, bradycardia, epistaxis, airway bleeding, excessive cough, transient laryngospasm, procedure induced atelectasis, pneumothorax and in rare cases, even fatalities [3-4].

\section{Cine-MRI versus cine-CT}

The most important advantage of cine-MRI compared with cine-CT was that it did not expose the children to ionising, which is a greater risk for children than adults [10-14]. Since cine-MRI does not require ionising radiation, all required breathing manoeuvres can be tested without concerns for radiation exposure. In addition, the procedure can be repeated when not executed correctly. In our group of patients, we had to repeat at least one acquisition per examination in $50 \%$ of tested children. Hence, this could be done without adding risks for the child.

\section{Feasibility of spirometer controlled cine-MRI}

Cine-MRI also has a number of limitations, the most important of which is the need for optimal cooperation by the patient. This is a clear challenge, especially in young children. Most children are able to do spirometry from the age of $\geqslant 6$ years, however, having a child enter the bore of the MRI covered by a body-coil, with a spirometer in their mouth (fig. 1c), followed by the requested breathing manoeuvres, requires careful preparation and coaching. In general we consider our spirometer controlled cine-MRI feasible for children aged $\geqslant 8$ years. In our small series we only had one child, aged 11 years, who could not complete the intended manoeuvres. 
The second limitation of our cine-MRI protocol was the low spatial resolution. Clearly, the resolution of the MRI images is inferior to that of CT [9]. In our MRI protocol we obtained an isotropic voxel of $3.0 \mathrm{~mm}^{3}$, which is inferior to the submillimetric voxels that can be achieved with CT [9]. However, this resolution was sufficient to detect the reduction of the airway lumen of the large airways, with diameters ranging from 1 to $2 \mathrm{~cm}$ in children 6-18 years [23]. For smaller airways and dynamic images a higher spatial resolution will be needed. We expect that by using parallel imaging, a dedicated torso coil and a higher field strength, the spatial and temporal resolution can be further improved in the near future.

\section{Further validation}

A major limitation of our retrospective study is that we evaluated a small selected group of patients. However, the main aim of our study was to test the feasibility of the method for later use on a larger scale with a more heterogeneous group of patients [24] Therefore, further validation of cine-MRI as a diagnostic method for TBM is required using a prospective study. We aim to do this comparing the diagnostic performance of bronchoscopy to spirometer controlled 3D cine-MRI.

\section{Conclusions}

This retrospective study showed that spirometer controlled 3D cine-MRI is a promising method to assess static and dynamic changes of central airway dimensions when TBM is suspected. Cine-MRI may be a more sensitive, faster and safer alternative for the diagnosis of static and dynamic TBM in cooperative children than bronchoscopy or cine-CT. Further prospective validation studies are required comparing the sensitivity and specificity of cine-MRI versus bronchoscopy and/or CT to diagnose TBM.

\section{References}

1 Carden KA, Boiselle PM, Waltz DA, et al. Tracheomalacia and tracheobronchomalacia in children and adults: an in-depth review. Chest 2005; 127: 984-1005.

2 Boogaard R, Huijsmans SH, Pijnenburg MWH, et al. Tracheomalacia and bronchomalacia in children: incidence and patient characteristics. Chest 2005; 128: 3391-3397.

3 de Blic J, Marchac V, Scheinmann P. Complications of flexible bronchoscopy in children: prospective study of 1,328 procedures. Eur Respir J 2002; 20: 1271-1276.

4 Midulla F, de Blic J, Barbato A, et al. Flexible endoscopy of paediatric airways. Eur Respir J 2003; 22: 698-708.

5 Williamson JP, James AL, Phillips MJ, et al. Quantifying tracheobronchial tree dimensions: methods, limitations and emerging techniques. Eur Respir J 2009; 34: 42-55.

6 Gilkeson RC, Ciancibello LM, Hejal RB, et al. Tracheobronchomalacia: dynamic airway evaluation with multidetector CT. AJR Am J Roentgenol 2001; 176: 205-210.

7 Baroni RH, Feller-Kopman D, Nishino M, et al. Tracheobronchomalacia: comparison between end-expiratory and dynamic expiratory CT for evaluation of central airway collapse. Radiology 2005; 235: 635-641.

8 Heyer CM, Nuesslein TG, Jung D, et al. Tracheobronchial anomalies and stenoses: detection with low-dose multidetector CT with virtual tracheobronchoscopy-comparison with flexible tracheobronchoscopy. Radiology 2007; 242: 542-549.

9 Lee EY, Boiselle PM. Tracheobronchomalacia in infants and children: multidetector CT evaluation. Radiology 2009; 252: 7-22.

10 de Jong PA, Mayo JR, Golmohammadi K, et al. Estimation of cancer mortality associated with repetitive computed tomography scanning. Am J Respir Crit Care Med 2006; 173: 199-203.

11 Kubo T, Lin PJ, Stiller W, et al. Radiation dose reduction in chest CT: a review. AJR Am J Roentgenol 2008; 190: 335-343.

12 Sadetzki S, Mandelzweig L. Childhood exposure to external ionising radiation and solid cancer risk. Br J Cancer 2009; 100: 1021-1025.

13 Strauss KJ, Goske MJ, Kaste SC, et al. Image gently: ten steps you can take to optimize image quality and lower CT dose for pediatric patients. AJR Am J Roentgenol 2010; 194: 868-873.

14 Brink JA, Amis ES. Image wisely: a campaign to increase awareness about adult radiation protection. Radiology 2010; 257: 601-602.

15 Eichinger M, Tetzlaff R, Puderbach M, et al. Proton magnetic resonance imaging for assessment of lung function and respiratory dynamics. Eur J Radiol 2007; 64: 329-334.

16 Yedururi S, Guillerman RP, Chung T, et al. Multimodality imaging of tracheobronchial disorders in children. Radiographics 2008; 28: e29.

17 Suto Y, Tanabe Y. Evaluation of tracheal collapsibility in patients with tracheomalacia using dynamic MR imaging during coughing. AJR Am J Roentgenol 1998; 171: 393-394.

18 Faust RA, Remley KB, Rimell FL. Real-time, cine magnetic resonance imaging for evaluation of the pediatric airway. Laryngoscope 2001; 111: 2187-2190.

19 Heussel CP, Ley S, Biedermann A, et al. Respiratory lumenal change of the pharynx and trachea in normal subjects and COPD patients: assessment by cine-MRI. Eur Radiol 2004; 14: 2188-2197.

20 Eichinger M, Puderbach M, Smith HJ, et al. Magnetic resonance-compatible-spirometry: principle, technical evaluation and application. Eur Respir J 2007; 30: 972-979.

21 Wielopolski PA, Ciet P, Krestin GP, et al. Automated airway lumen segmentation and characterization for use in patients with traqueomalacya: a feasibility Study. 2010. Proceedings of the ISMRM-ESMRMB Joint Annual Meeting, 2010. Abstract 4610. Available from: http://cds.ismrm.org/protected/10MProceedings/files/1_program. htm Date last accessed: November 4, 2013 
22 Turski PA, Korosec FR, Carroll TJ, et al. Contrast-enhanced magnetic resonance angiography of the carotid bifurcation using the time-resolved imaging of contrast kinetics (TRICKS) technique. Top Magn Reson Imaging 2001; 12: 175-181.

23 Griscom NT, Wohl ME. Dimensions of the growing trachea related to age and gender. AJR Am J Roentgenol 1986; 146: 233-237.

24 Thabane L, Ma J, Chu R, et al. A tutorial on pilot studies: the what, why and how. BMC Med Res Methodol 2010; $10: 1$. 